\title{
Cutting cardiovascular risk in barbershops
}

\section{Keywords}

Cardiovascular disease

Community-based health screening

African American populations
Deborah S. Main, PhD Department of Health and Behavioral Sciences, University of Colorado Denver, USA

Elizabeth M. Whitley, PhD, RN

Director, Denver Health Community Voices, Denver Health and Hospital Authority, USA

Pedro Arévalo Rincon, Program Manager, Denver Health Community Voices, Denver Health and Hospital Authority, USA

Joe McGloin, MA

Department of Family Medicine, University of Colorado Denver, USA

Mark A. Hocker Community Health Worker, Denver Health Community Voices, Denver Health and Hospital Authority, USA

Stanley K. Thomas Community Health Worker, Denver Health Community Voices, Denver Health and Hospital Authority, USA

Patti Iwasaki, MSW Department of Family Medicine, University of Colorado Denver, USA

E-mail:

Debbi.Main@ucdenver.edu

Online 28 October 2009

Deborah S. Main, Elizabeth M. Whitley, Pedro Arévalo Rincon, Joe McGloin, Mark A. Hocker, Stanley K. Thomas and Patti Iwasaki

Abstract

Background: Racial and ethnic minorities have lower cardiovascular disease (CVD) risk factor control and lower awareness of their CVD risk status despite being at higher risk for the disease. We sought to understand the reach and potential impact of delivering a CVD risk reduction program by Community Health Workers (CHW) in 15 Denver, Colorado barbershops.

Methods: This mixed-methods study analyzed data from CVD screenings of 1674 barbershop clients and from semi-structured interviews with barbershop owners.

Results: In 2 years, two CHWs screened a total of 1674 clients in barbershops for cardiovascular risk, nearly $80 \%$ of whom were African American. Screening results indicated that clients visiting barbershops are at risk for CVD and other health conditions. One third of clients had medium or high Framingham risk scores, one-quarter had mildly to severely high blood pressure, $45 \%$ had borderline high or high cholesterol, with $75 \%$ of clients considered overweight or obese according to BMI. Importantly, $41 \%$ of screened clients reported having no prior knowledge of their CVD risk before the screening. Barbershop owners reported many benefits of this screening program on their businesses, clients and personal health.

Conclusions: Barbershops represent a promising venue for reaching primarily male, African American populations at risk for cardiovascular disease. (c) 2009 WPMH gmbH. Published by Elsevier Ireland Ltd (c) 2009 WPMH GmbH. Published by Elsevier Ireland Ltd.

\section{Introduction}

Cardiovascular disease (CVD) remains the leading cause of death in the United States with death rates most pronounced among African Americans and men [1,2]. Modifiable risk factors, such as smoking, dyslipidemia, hypertension, overweight, obesity and physical inactivity are major contributors to CVD [3]. Although national guidelines have been established to prevent new cases of CVD, we have made little progress in meeting national health goals for blood pressure, heart disease and stroke. Fewer than half of people have their risk factors assessed, treated or controlled, and many are not aware that they have elevated risk for CVD [4] - with studies showing that racial and ethnic minorities have lower CVD risk factor control and lower awareness of their CVD risk status despite being at higher risk for the disease $[5,6]$.
The high prevalence of potentially modifiable risk factors provides great opportunities for the prevention of CVD in African American populations $[2,7,8]$, but only if we can identify better strategies for reaching this group. There is a growing body of research supporting the value of reaching this population through churches $[9,10]$, but less about another popular place for social connection among the African American male community: the barbershop. To date, limited research on the use of barbershops for reaching African American men with health-related programming is promising, with this setting being used to target programming on human immunodeficiency virus/sexually transmitted diseases (HIV/STDs), nutrition education, cancer screening, and CVD and risk reduction [11-13]. In New Orleans, barbers were trained to measure their clients' blood pressures and distribute health education 
material as part of the Healthy Heart Community Prevention project targeting African Americans in low socioeconomic neighborhoods. However, the researchers found this intervention to be difficult to maintain and sustain [14]. More recently, results from two feasibility studies conducted in Texas suggest that barbershops can be effective for hypertension detection, provider referral and follow-up [15]. Taken together, research to date highlights the potential value of barbershops as venues for promoting health among African American populations but more research is warranted about their benefit for clients and for barbers.

The purpose of the present study is to describe the preliminary findings of a 3-year project that employs trained Community Health Workers (CHW) in barbershops to screen and educate primarily African American men on CVD risk and risk reduction. We will briefly describe the quantitative results of our barbershop screenings in terms of populations we have reached and their CVD risk status. We also present data from semi-structured interviews with barbers on their perceptions of the screening program and its impact on their barbershops, clients and their personal health.

\section{Methods}

\section{Barbershop-Based CVD Screening Program}

The Barbershop screening program is part of a larger, 3-year Community Cardiovascular Disease Prevention Program grant funded through tobacco tax revenue awarded by the Colorado Department of Public Health and Environment. The goal of the program is that clients will become aware of their numbers (blood pressure, cholesterol, glucose, body mass index (BMI)), and their risk of developing CVD (using the Framingham risk score) [16-18]. Resources are offered to assist them in changing modifiable behaviors related to nutrition, physical activity and smoking. Additionally, individuals at moderate and high risk for CVD are identified and linked to primary care for treatment. We employed two, trained, Community Health Workers (CHWs) to provide screening, CVD risk assessment, and referrals to primary care and other community resources for primarily African American males visiting 15 barbershops in
Denver, Colorado, USA. A unique feature of the CVD screening program is that CHWs use pentablet computers to enter all screening and other client data. The HIPPA-compliant software system helps the CHWs manage their work by guiding them through the screening process, it also calculates a Framingham risk score, tracks both medical and community referral resources and tracks actual referrals made at the individual level. It creates follow up reminders as well as providing management reports so that CHW activities can be monitored. Barbershop owners provided space for the CHW to conduct screenings and to present educational sessions. Barbers were asked to encourage their clients to participate in the screening and to reinforce health messages about CVD risk reduction. They also served as role models for their clients as many participated themselves. Each participating business owner was given an incentive of $\$ 800$ per year, with barbers also recognized for participating in the program through signage as well as through the media.

\section{Content of CVD Screening Program}

The CHW visited each barbershop on a weekly basis. The CHW screened clients one at a time, spending an average of 14 minutes with each person. The CHW informed each interested client about the screening process, that their screening results would be confidential and that only de-identified data would be reported. Figure 1 describes the CVD screening process. Client-specific data were collected using clinical screening data on non-fasting cholesterol, blood pressure and BMI (from measured height and weight) and self-reported data on family history of several chronic conditions, physical activity, healthy eating, tobacco, and presence of CVD. (Note: we added blood glucose only in the last year of the program so that information is not reported in this paper). These data were used to identify CVD risk factors (overweight, inactivity, smoking, and hypertension) and to calculate an overall Framingham risk score. CHWs also collected data on whether clients knew their risk status prior to the screening/assessment. Screening and risk information was shared with clients on a Prescription for Good Health card. The CHW also entered data on referrals to primary care and community resources, and worked with the client in developing a plan for follow-up. 


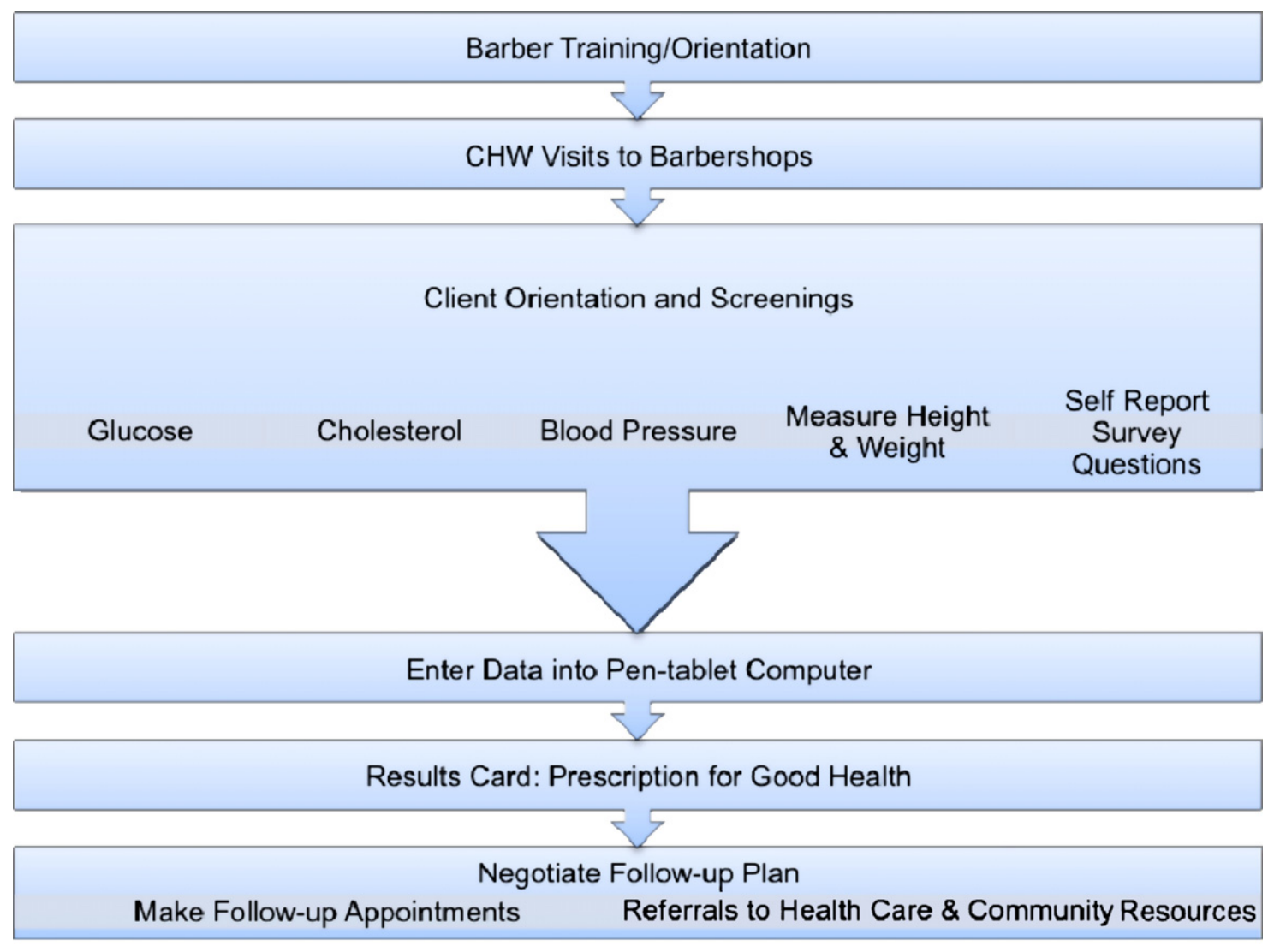

Figure 1 Barbershop cardiovascular disease (CVD) screening process.

\section{Data Collection to Evaluate Program Reach and Potential Impact}

During the course of the first 2 years of the project, we collected data using pen-tablet computers on 1674 screened clients in 15 barbershops, and conducted semi-structured interviews with 14 of 15 barbershop owners to determine their perceptions of the screening program and its impact on clients, their business and their personal health. We report the initial descriptive findings of our mixed methods analysis from these first 2 years, focusing primarily on CVD risk of screened clients and barber reactions to this new CVD screening program.

\section{Results}

Through both quantitative screening data and qualitative interviews we have collected useful information about the reach of our screening programs, the CVD risk of screened clients, and how barbershop-based screening programs have been received by, and affected, barbershop owners, barbers, and clients. This information is briefly summarized below.

\section{Screening Results}

Over the course of 2 years, the two CHWs screened a total of 1674 people in 15 barbershops. As shown in Table 1, nearly two-thirds of clients screened were male $(63 \%)$ and over three-quarters were African American (79\%). Interestingly, the screened population was fairly young, with only 35\% being over the age of 50 . Over one-quarter $(28 \%)$ of participants reported having no health insurance, and $24 \%$ did not have a medical home.

Table 2 illustrates the risk status of screened clients overall and broken down by gender. The screening results indicate that clients visiting barbershops are indeed at risk for CVD and other health conditions. One quarter (25\%) had a medium and 9\% a high Framingham risk score (i.e., risk for CVD in next 10 years), with male clients having higher Framingham risk scores than female clients. In terms of modifiable risk factors, over a quarter of screened clients were current smokers (28\%), with men 
Table 1 Demographic profile of 1674 clients screened in barbershops

\begin{tabular}{ll}
\hline Demographics & $\%$ \\
\hline Age & \\
Under 35 & 29 \\
$35-50$ & 35 \\
Over 50 & 35 \\
Gender & \\
Male & 63 \\
Female & 37 \\
Race/Ethnicity & \\
African American & 79 \\
White & 5 \\
Hispanic/Latino & 9 \\
Other & 7 \\
Insurance status & \\
Insured & \\
Uninsured & 72 \\
Have medical home & 28 \\
Yes & \\
No & 76 \\
\hline
\end{tabular}

more likely to report smoking (30\%) than women $(25 \%)$. Over one quarter of clients had blood pressure above the normal range ( $17 \%$ considered to have mildly high, $9 \%$ moderately high and $1 \%$ severely high blood pressure), with patterns of high blood pressure being fairly similar for men and women. Furthermore, only 25\% were considered to be of a healthy weight overall $(40 \%$ were overweight and $34 \%$ obese) according to BMI measures - with similar levels of unhealthy weight among men and women (although with different distributions of overweight and obesity). Finally, 55\% were found to have desirable, 36\% borderline high, and 9\% high cholesterol levels, with patterns being similar among men and women.

Of particular interest, $41 \%$ of screened clients reported having no knowledge of their CVD risk before the screening (36\% men versus $50 \%$ women) - a finding that supports the value of using CHWs to screen, and educate, clients about risk and risk reduction in com-

Table 2 CVD risk status of 1674 clients screened in barbershops

\begin{tabular}{|c|c|c|c|}
\hline Risk Status & $\begin{array}{l}\% \text { Male } \\
(n=1062\end{array}$ & $\begin{array}{l}\% \text { Female } \\
(n=612)\end{array}$ & $\begin{array}{l}\text { Total \% } \\
(n=1674\end{array}$ \\
\hline Family history of heart disease & 25 & 22 & 24 \\
\hline Family history of diabetes & 35 & 28 & 32 \\
\hline Currently smoke cigarettes & 30 & 25 & 28 \\
\hline \multicolumn{4}{|l|}{ Total cholesterol } \\
\hline Desirable & 53 & 58 & 55 \\
\hline Borderline & 35 & 34 & 36 \\
\hline High & 12 & 8 & 9 \\
\hline \multicolumn{4}{|l|}{ Blood pressure/hypertension } \\
\hline None & 71 & 76 & 74 \\
\hline Mild & 19 & 14 & 17 \\
\hline Moderate & 9 & 8 & 9 \\
\hline Severe & 1 & 2 & 1 \\
\hline \multicolumn{4}{|l|}{ Framingham risk score } \\
\hline Low & 59 & 80 & 67 \\
\hline Medium & 29 & 18 & 25 \\
\hline High & 12 & 2 & 9 \\
\hline \multicolumn{4}{|l|}{$\mathrm{BMI}$} \\
\hline Underweight & 1 & 3 & 2 \\
\hline Healthy weight & 25 & 23 & 25 \\
\hline Overweight & 42 & 34 & 40 \\
\hline Obese & 32 & 40 & 34 \\
\hline \multicolumn{4}{|l|}{ Knowledge of risk } \\
\hline Yes & 64 & 50 & 59 \\
\hline No & 36 & 50 & 41 \\
\hline
\end{tabular}


munity screenings like barbershops. Importantly, our findings indicate that these screenings went beyond information and education: CHWs referred 279 clients to health and health care resources in the Denver area (e.g., QuitLine), and had over 200 follow-up visits with the same clients over time.

\section{Summary of Barbershop Owner Interviews}

Interviews with barbershop owners (10 out of 14 of whom were male) revealed interesting and important information about the perceived value of having a barbershop-based CVD screening program. Barbershop owners supported the value of having screening programs in these settings and of having a CHW present on an ongoing basis. Most talked about the barbershop as a good place to reach men who do not go to, or trust, doctors. Furthermore, in some cases these men encouraged other friends and family members to come back and get screened. Barbershop owners acknowledged the powerful role that barbershops can and do play in the lives of their clients. Most barbershop owners felt the program was going well and all felt it was beneficial on many different levels. For example, many owners noted changes in their shops and among the clients since the screening program began; several noted that the make-up of their clientele changed as a result of the program. They also talked about having made personal lifestyle changes in three main areas: reading more about heart disease, exercising more, and changing cooking and eating habits. None of the barbershop owners could cite or even think of a negative aspect of the program. The overwhelming majority felt the program was making a very big difference in the lives of their patrons.

\section{Discussion and Conclusion}

Barbershops are effective venues for reaching African Americans who are at risk, and, often unaware of their risk of CVD. This innovative Barbershop CVD screening program is a replicable model for community-based screening, CVD risk assessment and referral to primary care among clients in barbershops. Our program has established the value of using CHWs for this purpose, adding to a growing literature on the positive impact of CHWs on the health of diverse populations with hypertension and other chronic conditions [19-22]. A key to our outreach is that we employ culturally competent workers able to build trust and rapport with both barbers and their clients - critical to delivering programs in this setting in efforts to reduce health disparities $[23,24]$. These trusted relationships take some time to establish, but they lend tremendous credibility to the value of CVD screening and risk reduction in this population, the need for ongoing support and encouragement by $\mathrm{CHWs}$ and barbers, and the added benefit of having these programs embedded within an important place where African American men congregate.

There are several limitations to the present findings. First, we have reported only the first 2 years of data from a 3-year project - additional data will help to determine the stability of our initial findings and whether barbershop patrons screened look similar over time. Furthermore, although our program does include a follow-up component (particularly for those found to be at risk), the relatively low frequency and intensity of follow-up visits are not expected to result in demonstrable changes in "numbers" over time. We have strengthened the follow-up component in our current program and will be more aggressively linking clients to health care and other community resources and tracking them over time to determine impact. Also, we report only data from those people who agreed to be screened in barbershops - we do not know whether and how our sample differs from those who do not seek screening or from the overall population of people who patronize these 15 barbershops. Finally, it is important to note that clinical values resulting from these community screenings are not intended to be diagnostic, hence our descriptive findings from these clinical and self-report data should be interpreted with caution.

The 2-year findings we have described in this paper contribute to the existing public health literature by demonstrating the success we have had in reaching primarily African American men and the novel use of pen-tablet computers by CHWs for screening, referral and tracking barbershop clients over time. The literature on conducting CVD screening 
in barbershops is scarce, and, to date, there are no randomized controlled trials demonstrating impacts on health outcomes. Of note, Hess et al. found that barbers can be effective in actually measuring and monitoring blood pressures among hypertensive clients, while the model we describe employs CHWs to screen and educate clients and deploys barbers primarily as peer educators and role models $[13,15]$. Further research is needed on barbershop-based interventions with African Americans to evaluate their efficacy and cost effectiveness in detecting and reducing CVD risk.

\section{Acknowledgements}

This program was funded by the Cancer, Cardiovascular Disease and Pulmonary Disease Grants Program, at the Colorado Department of Public Health and Environment. Much appreciation to the Barbers from the participating Barbershops for their contributions.
References

[1] Centers for Disease Control and Prevention. The Burden of Chronic Diseases and Their Risk Factors: National and State Perspectives 2004. Atlanta, GA: CDC; 2004. Available at: http://www.cdc.gov/nccdphp/ burdenbook2004. (Accessed July 28, 2008).

[2] Clark LT. Issues in minority health: atherosclerosis and coronary heart disease in African Americans. Med Clin North Am 2005;89:977-1001.

[3] Yusef S, Hawken S, Ounpuu S, Dans T, Avezum A, Lanas F, et al. Effect of potentially modifiable risk factors associated with myocardial infarction in 52 countries (the INTERHEART study): case control study. Lancet 2004;364:937-52.

[4] Greenland P, Knoll MD, Stamler J, Neaton $J D$, Dyer AR, Garside DB, et al. Major risk factors as antecedents of fatal and nonfatal coronary heart disease events. JAMA 2003;290:891-7.

[5] Department of Public Health and Environment. Health Disparities and Cardiovascular Disease. Denver, CO: Colorado Department of Public Health and Environment; 2005.

[6] Kurian AK, Cardarelli KM. Racial and ethnic differences in cardiovascular disease risk factors: a systemic review. Ethn Dis 2007; 12:143-52.

[7] Watson KE. Cardiovascular risk reduction among African Americans: a call to action. J Natl Med Assoc 2008;100:18-26.

[8] Clark LT, Ferdinand KC, Flack JM, Gavin 3rd JR, Hall WD, Kumanyika SK, et al. Coronary heart disease in African Americans. Heart Dis 2001;3:97-108.

[9] Campbell MK, Demark-Wahnefried W, Symons M, Kalsbeek WD, Dodds J, Cowan $A$, et al. Fruit and vegetable consumption and prevention of cancer: the Black churches united for better health project. Am J Public Health 1999;89:1390-6.

[10] Yanek LR, Becker DM, Moy TF, Gittelsohn J, Koffman DM. Project Joy: faith based cardiovascular health promotion for African American women. Public Health Rep 2001;116(Suppl 1):68-81.

[11] Magnus M. Barbershop nutrition education. J Nutr Educ Behav 2004;36:45-6.

[12] Lewis YR, Shain L, Quinn SC, Turner K, Moore T. Building community trust: lessons from an STD/HIV peer educator program with African American Barbers and Beauticians. Health 2002;3(2):133-43.

[13] Hess PL, Reingold JS, Ruger E, Clark S, Griffin GW, Leonard D, et al. Efficacy of a barbershop-based intervention to improve the treatment and control of hypertension in African American men. Am J Hypertens 2004;17:198A-9A [Abstract P-451].

[14] Ferdinand $K$. Lessons learned from the healthy heart community prevention project in reaching the African American population. J Health Care Poor Underserved 1997;3:366-71.

[15] Hess PL, Reingold JS, Jones J, Fellman MA, Knowles P, Ravenell JE, et al. Barbershops as hypertension detection, referral and followup centers for black men. Hypertension 2007;49:1040-6.

[16] Grundy SM, Balady GJ, Criqui MH, Fletcher G, Greenland P, Hiratzka LF, et al. Primary prevention of coronary heart disease: guidance from Framingham: a statement for healthcare professionals from the AHA Task Force on Risk Reduction American Heart Association. Circulation 1998;97(18):187687.

[17] Wilson P, D'Agostino R, Levy D, Belanger $A M$, Silbershatz $H$, Kannel WB. Prediction of coronary heart disease using risk factor categories. Circulation 1998;97:1837-47.

[18] Grundy S, Pasternak R, Greenland P, Smith Jr S, Fuster V. Assessment of cardiovascular risk by use of multiple risk factor assessment equations: a statement for healthcare professionals from the American Heart Association and the American College of Cardiology. Circulation 1999;100:1481-92.

[19] Fedder D, Chang R, Curry S, Nichols G. The effectiveness of a community health worker program on healthcare utilization of west Baltimore City Medicaid patients with or without hypertension. Ethn Dis 2003;13:22-7.

[20] Morisky D, Lees N, Sharif C, Liu KY, Ward HJ. Reducing disparities in hypertension control - a community-based hypertension control project for an ethnically diverse population. Health Promotion Pract 2002;3:264-75.

[21] Dennison C, Hill MN, Bone LR, Levine DM. Comprehensive hypertension care in underserved urban black men - high follow-up rates and blood pressure improvement over 60 months. Circulation 2003;108(17: IV-381.

[22] Levine D, Bone L, Hill M, Stallings R, Gelber $A C$, Barker $A$, et al. The effectiveness of a community academic health center partnership to decrease the level of blood pressure in an urban African-American population. Ethn Dis 2003;13:354-61.

[23] Alcalay R, Alvarado M, Balcazar H, Newman E, Huerta E. Salud para su corazon: a community-based Latino cardiovascular disease prevention and outreach model. J Community Health 1999;24(5):359-79.

[24] Levine D, Becker D, Bone L. Narrowing the gap in health status of minority populations: a community-academic medical center partnership. Am J Prev Med 1992;5:321. 